\title{
Análise dos transientes da fluorescência da clorofila $a$ de plantas jovens de Carapa guianensis e de Dipteryx odorata submetidas a dois ambientes de luz
}

\author{
José Francisco de Carvalho GONÇALVES ${ }^{1}$, Carlos Eduardo SILVA², Diogo Gato GUIMARÁES³ ${ }^{3}$ Regiane \\ Sablina BERNARDES ${ }^{4}$
}

\begin{abstract}
RESUMO
Espécies arbóreas tropicais estáo quase sempre sob alta irradiância na Amazônia. O elevado fluxo energético pode ser decisivo para o sucesso ou fracasso no estabelecimento inicial das plantas no campo. O objetivo deste trabalho foi investigar as respostas de plantas jovens de andiroba (Carapa guianensis Aubl) e cumaru (Dipteryx odorata (Aublet) Willd) expostas a dois ambientes de luz (sombra e sol). O experimento foi conduzido em Manaus-AM, Brasil (3ㅇ'S, 59 $52^{\prime} \mathrm{W}$ ). As análises dos transientes da fluorescência da clorofila $a$ e os parâmetros relacionados foram obtidos em folhas intactas e saudáveis no período entre 10:00 e 12:00h. A curva OJIP indicou que ambas as espécies expostas à alta irradiância apresentaram fotoinibição após 45 dias de experimento. Plantas de andiroba e cumaru expostas ao sol exibiram menores valores de TR/ABS (7,17 e 20,4\%, respectivamente), $\mathrm{ET} / \mathrm{TR}$ (14,5 e 27,2\%, respectivamente) e $\mathrm{ET} / \mathrm{ABS}$ (20,6 e 42,2\%, respectivamente) e maiores valores de $\mathrm{DI} / \mathrm{ABS}$ (26,9 e 43,0\%, respectivamente), comparadas com plantas exposta à sombra. Quanto ao fluxo fenomenológico, para a maioria dos parâmetros, menores valores foram observados para ambas as espécies expostas ao sol. Plantas de andiroba e cumaru expostas ao sol exibiram valores menores de $\mathrm{PI}_{\mathrm{ABS}}(63,3 \mathrm{e} 78,7 \%$, respectivamente) comparadas com plantas expostas à sombra no final do experimento. Apesar de ambas as espécies terem sofrido com o estresse por alta irradiância, plantas de andiroba apresentaram melhores desempenhos do que plantas de cumaru, sendo, portanto, melhor indicadas para uso em plantio de produção ou de recuperação de áreas degradadas.
\end{abstract}

palavras-Chave: Curva O-J-I-P, Fluxo fenomenológico, Índice de desempenho, Fotoinibição

\section{Analysis of chlorophyll a fluorescence transients of young plants of Carapa guianensis and Dipteryx odorata submitted to two light environments}

\begin{abstract}
Tropical tree species are almost always under high irradiance in the Amazonian. The high energy flux can be decisive for the success or not in the initial establishment in the field. The objective of this study was to investigate the answers of young plants of andiroba (Carapa guianensis Aubl) and cumaru (Dipteryx odorata (Aublet) Willd) exposed to two light environments (shade and sunlight). The experiment was carried in Manaus-AM, Brazil (3ㅇ's, 59 $\left.52^{\prime} \mathrm{W}\right)$. Analysis of chlorophyll a fluorescence transients were obtained in intact and healthy leaves between 10:00 to 12:00 am. The curve OJIP indicated that the species high irradiance exposed presented photoinhibition after 45 days of experiment. Andiroba and cumaru plants exposed to sunlight exhibited low values of TR /ABS (7.17 and 20.4\%, respectively), ET/TR (14.5 and 27.2\%, respectively) and ET/ABS (20.6 and $42.2 \%$, respectively) and high values of DI / ABS (26.9 and 43.0\%, respectively), compared with plants exposed to the shadow with 45 days of experiment. As for phenomenological flux, most of the parameters, low values were observed for both exposed species in the sunlight. Andiroba and cumaru plants exposed in the sunlight exhibited low values of $\mathrm{PI}_{\mathrm{ABS}}(63.3$ and $78.7 \%$, respectively) compared with exposed plants to the shadow at the end of the experiment. Although the species have suffered with the stress for high irradiance andiroba specie presented better performance than cumaru being indicated for use in production planting or degraded area restoration.
\end{abstract}

KEYWORDS: O-J-I-P curve, Phenomenological fluxes, Performance index, Photoinhibition

\footnotetext{
1 Instituto Nacional de Pesquisas na Amazonia - INPA, E-mail: jfc@inpa.gov.br

2 Instituto Nacional de Pesquisas na Amazonia - INPA, E-mail: cems@inpa.gov.br

3 Instituto Nacional de Pesquisas na Amazonia - INPA, E-mail: diogo_gato44@hotmail.com

${ }^{4}$ Instituto Nacional de Pesquisas na Amazonia - INPA, E-mail: sablina@hotmail.com
} 


\section{INTRODUÇÃO}

As plantas, necessariamente, dependem da energia solar para sobreviverem. Contudo, por mais importante que seja e por quantos benefícios a irradiância possa trazer para o bom desempenho fotossintético das plantas, mudanças abruptas na intensidade luminosa, como normalmente ocorrem em clareiras formadas nas florestas por açôes antrópicas ou por efeitos naturais e, até mesmo, em plantios comerciais utilizando espécies sucessionais tardias para a produção florestal, podem exceder o limite de tolerância das plantas ao fluxo de energia (Denslow et al., 1990; Tonini et al., 2008). Em geral, as plantas de estádios intermediários ou tardios na sucessão, quando na fase juvenil, crescem em ambientes de menor disponibilidade luminosa e os impactos das mudanças no ambiente de luz podem trazer conseqüências danosas para as mesmas. O fato é que, alta irradiância quando associada a outros fatores estressantes podem determinar a redução do crescimento ou até mesmo levar a morte da planta (DemmigAdams \& Adams, 1992).

A adaptação do aparato fotossintético para diferentes densidades de fluxos fotossintéticos, geralmente, passa por ajustamentos de proporçôes relativas dos componentes que envolvem a absorção, a transmissão e a utilização da energia luminosa, na qual a quantidade da energia absorvida é inerente à capacidade de uso do produto da reaçáo fotoquímica, ou seja, quando esse balanço é desalinhado proporciona excesso na capacidade fotoquímica favorecendo o surgimento de danos às vezes irreversíveis para a planta (Walters, 2005). Assim, plantas que crescem em ambientes sombreados e são expostas à alta irradiância podem sofrer fotoinibição ou danos irreparáveis no aparato fotossintético (Long et al., 1994; Park et al., 1996).

Quando as plantas se ajustam aos danos fotoinibitórios causados pela transferência para ambientes com alta irradiância, estas iniciam mudanças morfofisiológicas para uma adequação ao novo ambiente. Tais mudanças são referidas como aclimatação (Walters, 2005). Existe grande número de plantas com potencial para ajuste em diversos regimes de irradiância. Estas alteraçôes morfo-anatômicas e fisiológicas ocorrem mais rapidamente em folhas de espécies de plantas herbáceas. Porém, algumas plantas de espécies arbóreas tropicais, no estádio juvenil, são extremamente lentas neste ajustamento (Langenheim et al., 1984).

Estudos sobre exposição ao excesso de irradiância indicam que as plantas experimentam diminuiçáo na eficiência do fotossistema II, com conseqüente mudança no direcionamento da energia fotoquímica por meio da dissipação (Castro et al., 1995; Demmig-Adams et al., 1996; Santos Jr. et al, 2006; Gonçalves et al., 2007). Recentemente, tais investigaçóes têm sido realizadas com o uso de novas ferramentas que ajudam a elucidar o comportamento de plantas quando submetidas a situaçóes de estresse. O transiente OJIP é uma ferramenta de análise da mudança na cinética da fluorescência da clorofila $a$ que fornece informaçôes detalhadas sobre a estrutura e função do aparato fotossintético, especialmente do fotossistema II (Lazár, 2006). Contudo, pouco se conhece sobre o uso dessa ferramenta ecofisiológica, no que se refere ao comportamento de plantas arbóreas tropicais jovens, quando submetidas a diferentes intensidades de irradiância, principalmente, espécies como $C$. guianensis e $D$. odorata, ambas sucessionais tardias, conforme Boufleuer (2004) e Lorenzi (1998), respectivamente. Diante disto, a expectativa é que estas espécies sejam sensíveis à elevada irradiância quando jovem e, como conseqüência, possam apresentar dificuldades para serem cultivadas. Por outro lado, são espécies que apresentam elevado valor econômico e com potencial utilização em plantios para reflorestamentos e produçáo florestal (Clay et al., 2000; Tonini et al., 2008). Portanto, o conhecimento da resposta da fluorescência da clorofila $a$ de plantas jovens de C. guianensis e D. odorata representará importante ganho, para que na prática, seja feita a seleção de espécies/indivíduos aptos a povoarem áreas de plantios ou utilização dessas plantas em programas de reflorestamento sobre áreas degradadas na Amazônia. O objetivo deste estudo foi analisar os transientes da fluorescência da clorofila $a$ de plantas jovens das espécies C. guianensis e $D$. odorata em resposta a diferentes níveis de irradiância, visando avaliar o desempenho das mesmas sob condiçôes de baixa e alta disponibilidade de luz.

\section{MATERIAL E MÉTODOS}

\section{LOCAL DE ESTUDO}

O trabalho foi realizado no campus experimental do Instituto Nacional de Pesquisas da Amazônia - MCT/INPA, Manaus, AM, Brasil ( $3^{\circ} 8^{\prime} \mathrm{S}$ e $\left.59^{\circ} 52^{\prime} \mathrm{W}\right)$, onde, plantas jovens (90 dias) de C. guianensis (Carapa guianensis Aubl) e D. odorata (Dipteryx odorata (Aublet) Willd), oriundas de matrizes da Reserva Florestal Adolpho Ducke, localizada a $10 \mathrm{~km} \mathrm{da}$ cidade de Manaus, e apresentando uniformidade de tamanho (aproximadamente $35 \mathrm{~cm}$ de altura), foram transplantadas para sacos plásticos $(30 \times 25 \mathrm{~cm})$ com capacidade para $8 \mathrm{~kg}$, contendo mistura de terriço vegetal e argila na proporção de 3:1. Dez plantas de cada espécie foram selecionadas para compor o experimento. Durante 20 dias as plantas foram colocadas numa estrutura feita de tubos de pvc $(2 \times 6 \mathrm{~m})$ cercada por todos os lados utilizando sombrite de polietileno (50\%). As plantas foram irrigadas diariamente às 8:00 $\mathrm{h}$ da manhã até a capacidade de campo. Após o período de aclimatação (20 dias), dez plantas foram separadas aleatoriamente em dois tratamentos: 1) plantas expostas à sombra (permaneceram no sombrite, máxima irradiância de $100 \mu \mathrm{mol} \mathrm{m}^{-2} \mathrm{~s}^{-1}$ ); e 2) plantas expostas ao sol (máxima irradiância de $2500 \mu \mathrm{mol} \mathrm{m}^{-2}$ $\left.\mathrm{s}^{-1}\right)$. Para os diferentes tratamentos foram feitas determinaçôes 
nas plantas de parâmetros relacionados aos transientes da fluorescência da clorofila $a$. As determinaçóes foram feitas no tempo zero (leitura inicial) e em intervalos de 15 dias durante todo o período do experimento (45 dias). Contudo, alguns parâmetros foram melhor interpretados analisando-se apenas a leitura inicial e final, enquanto que outros foram bem explicados exibindo os resultados com intervalos de 15 dias.

\section{DETERMINAÇÃO DOS TRANSIENTES DA FLUORESCÊNCIA DA CLOROFILA A}

As medidas dos parâmetros relacionados à fluorescência da clorofila $a$ foram feitas em folhas apresentando bom estado fitossanitário e completamente expandidas (maduras), utilizando-se um fluorômetro portátil (Handy PEA - v 1.30 Hansatech Instruments, Norfolk, UK), entre 10:00 e 12:00 $\mathrm{h}$ do dia. As folhas selecionadas foram adaptadas ao escuro durante um período de 30 minutos, utilizando-se clipes apropriados. Após a adaptação, as folhas foram expostas a um pulso saturante de luz $\left(3000 \mu \mathrm{mol} \mathrm{m} \mathrm{m}^{-2} \mathrm{~s}^{-1}\right.$, comprimento de onda de $650 \mathrm{~nm}$, por 1 segundo), obtendo-se as respostas relacionadas aos transientes da fluorescência da clorofila $a$, de acordo com equações do teste OJIP (Strasser et al., 1995). O estádio inicial da atividade fotossintética no centro de reação é regulado por três etapas funcionais básicas e, portanto, são descritas de forma a explicar as variáveis da absorção do fóton pela molécula de clorofila no complexo antena (ABS); acúmulo de energia absorvida (TR); conversão da energia de excitação para a cadeia transportadora de elétrons (ET); além disso, considera-se a energia que é dissipada em forma de calor ou fluorescência (DI). Assim, foram obtidos os parâmetros do fluxo fenomenológico: $\mathrm{RC} / \mathrm{CS}$ = densidade de centros de reação por seção transversal; $\mathrm{ABS} / \mathrm{CS}$ = número de fotóns absorvidos por seção transversal; DI $/ C S$ = energia de dissipação por seção transversal; TR/CS = taxa máxima de energia bloqueada por seção transversal e; $\mathrm{ET}_{\mathrm{o}} / \mathrm{CS}$ = transporte de elétrons por seção transversal, bem como, os parâmetros das razóes de fluxo: TR/ABS = máxima eficiência quântica do fotossistema II (FSII); DI /ABS = máxima eficiência de excitação não fotoquímica; $\mathrm{ET}_{\mathrm{o}} / \mathrm{TR}_{\mathrm{o}}=$ probabilidade $\mathrm{da}$ energia de excitação mover um elétron após a quinona $\mathrm{A}$ e; $\mathrm{ET}_{\mathrm{o}} / \mathrm{ABS}$ = probabilidade de um fóton absorvido mover um elétron após $Q_{A}$. Além disso, foram obtidos, também, os valores referente a área da curva OJIP (Área), fluorescência inicial $\left(\mathrm{F}_{\mathrm{o}}\right)$, fluorescência variável $\left(\mathrm{F}_{\mathrm{v}}\right)$, fluorescência máxima $\left(\mathrm{F}_{\mathrm{m}}\right)$, tempo para alcance de $\mathrm{F}_{\mathrm{m}}\left(\mathrm{t}_{\mathrm{Fm}}\right)$, eficiência máxima do fotossistema II $\left(\mathrm{F}_{\mathrm{v}} / \mathrm{F}_{\mathrm{m}}\right)$ e ao índice de desempenho $\left(\mathrm{IP}_{\mathrm{ABS}}\right)$.

\section{DELINEAMENTO EXPERIMENTAL E ANÁLISE ESTATÍSTICA}

O delineamento experimental utilizado foi o inteiramente casualizado, utilizando-se duas espécies (C. guianensis e $D$. odorata) em dois ambientes de luz (sombra e sol), com cinco repetiçóes, considerando cada planta como uma repetição. Os resultados obtidos foram submetidos ao teste de normalidade Lilliefors, análise de variância - ANOVA e comparados pelo teste $t$ de Student $(P<0,05)$. O programa utilizado foi o JMP 4.0 (SAS Institute Inc., 2000).

\section{RESULTADOS E DISCUSSÃO}

\section{ANÁLISE DOS TRANSIENTES DA FLUORESCÊNCIA DA CLOROFILA A}

As duas espécies estudadas exibiram típica curva do transiente OJIP da fluorescência da clorofila $a$ nas leituras iniciais (Figura 1). Contudo, a área acima da curva de fluorescência entre $\mathrm{F}_{\mathrm{o}}$ e $\mathrm{F}_{\mathrm{m}}$ foi menor para a espécie $D$. odorata em relação à espécie ${ }^{C}$. guianensis, indicando que $C$. guianensis apresentou maior estoque de aceptores de elétrons do fotossistema II devido ao desbalanço entre o fluxo de elétrons do lado doador $\left(\mathrm{Y}_{z}\right)$ para o centro de reação $(\mathrm{RC})$ e o fluxo de elétrons do $\mathrm{RC}$ para o lado aceptor $\left(\mathrm{Q}_{\mathrm{A}}\right)$ com consequente diminuição da fluorescência máxima $\left(\mathrm{F}_{\mathrm{m}}\right)$ (Strasser, 1997) (Tabela 1). Quando se observou os transientes OJIP das plantas expostas ao sol (45 dias de experimento), verificou-se redução na elevação da curva para as duas espécies, quando comparada com o transiente OJIP inicial por conseqüência da redução dos valores de $\mathrm{F}_{\mathrm{m}}$. Quando se compararam os mesmos transientes OJIP para as duas espécies (exposta ao sol), verificou-se que $D$. odorata apresentou área acima da curva de fluorescência 2,5 menor, quando comparada com área para a espécie $C$. guianensis (Figura 1). Adicionalmente, o tempo para alcançar a fluorescência máxima $\left(\mathrm{t}_{\mathrm{Fm}}\right)$ foi menor para a espécie C. guianensis com 45 dias de exposição ao sol indicando melhor eficiência na transferência de elétrons ao longo do transiente OJIP (Tabela 1). Os valores da fluorescência variável relativa mostraram que a espécie $D$. odorata sofreu fortemente o estresse por alta incidência de irradiância, uma vez que a forma do transiente na fase J das plantas exposta ao sol foi mais pronunciada, em relação às plantas da sombra e, também, quando comparada com a espécie $C$. guianensis sob mesma condição de fluxo de energia (Figura 2). Quando se observou os dados normalizados entre $\mathrm{F}_{\mathrm{O}}$ e $\mathrm{F}_{\mathrm{J}}$ verificou-se desvio positivo para as duas espécies indicando claramente o efeito do estresse por alta incidência de irradiância (Figura 3).

A elevada irradiância provocou, nas plantas exposta ao sol, modificação no transiente OJIP com conseqüente diminuição dos valores de $\mathrm{F}_{\mathrm{m}}$, conforme observado em plantas de copaiba (Copaifera multijuga Hayne) e de mogno (Swietenia macrophylla King) crescendo sob dois ambientes de irradiância (Gonçalves et al., 2007). Normalmente, quando as plantas estão sob ambientes estressantes, o tipo de estresse não é claramente identificado pelo transiente da fluorescência da clorofila $a$ quando os dados são apresentados na forma linear, contudo, quando os valores do transiente OJIP são apresentados na forma variável relativa verifica-se, 

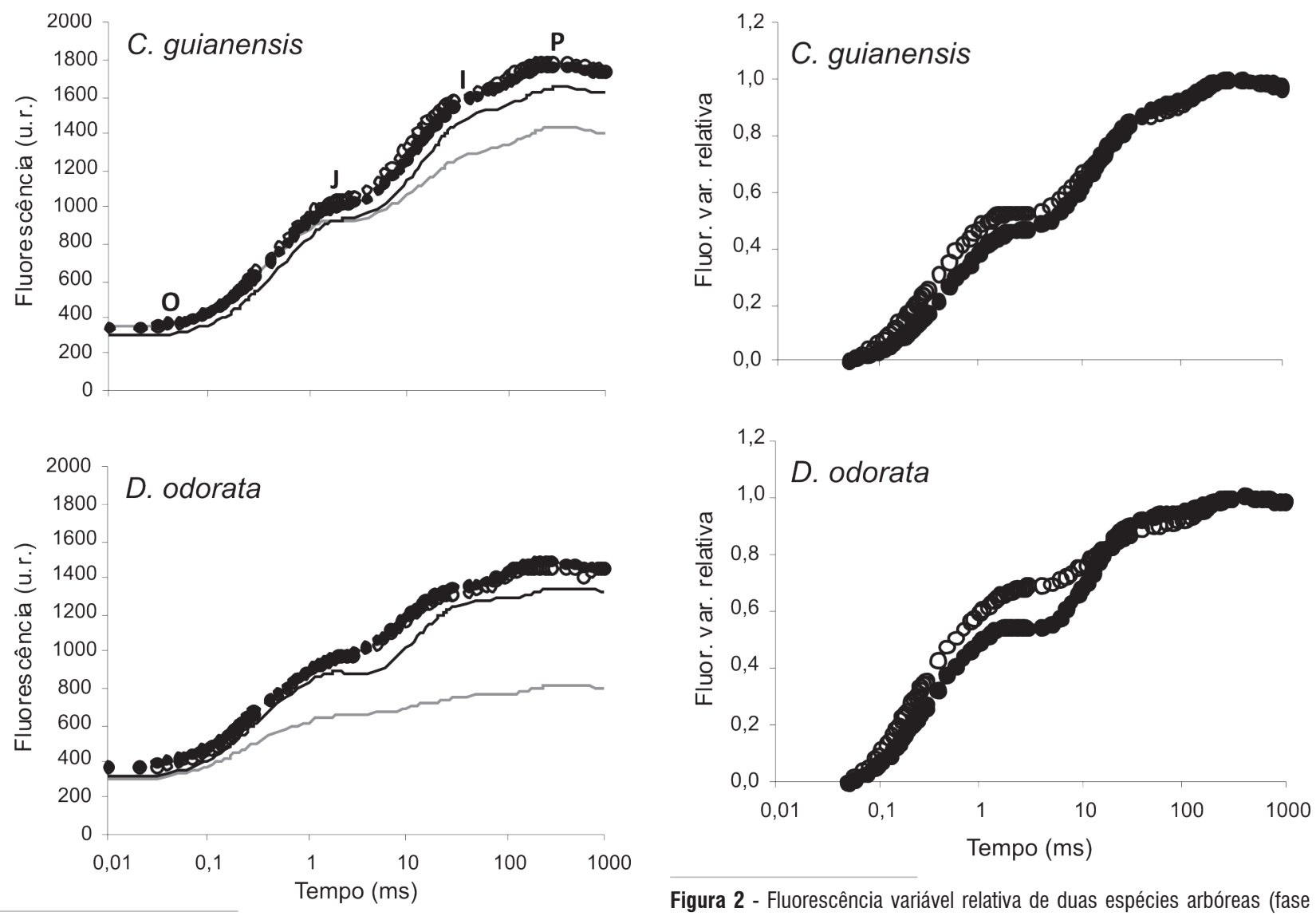

Figura 1 - Transiente da fluorescência da clorofila a de duas espécies arbóreas (fase juvenil) em dois ambientes de luz (sombra e sol). Valores médios das leitura iniciais dos indivídos nos tratamentos de sombra $(\bullet)$ e de sol $(\circ)$. Leitura final (45 dias de experimento) dos indivíduos nos tratamentos de sombra $(-)$ e de sol $(-)(n=5)$.

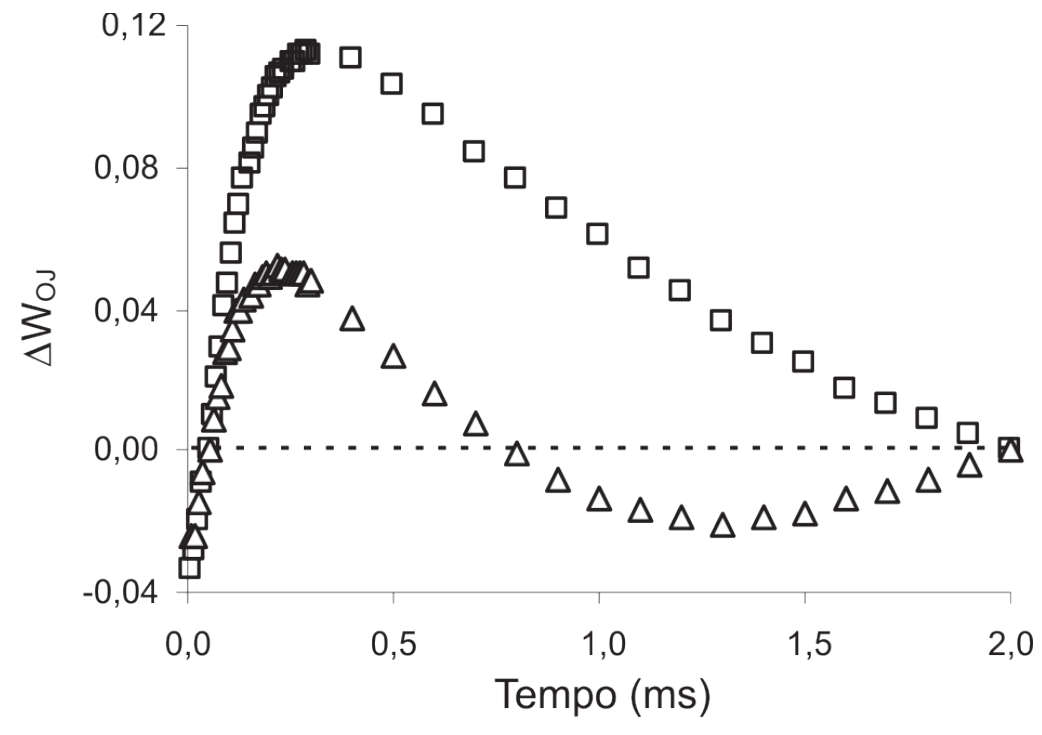

Figura 3 - Mudança na forma da curva do transiente da fluorescência da clorofila a normalizada entre 0 e J $\left(\Delta \mathrm{W}_{0 \mathrm{~J}}=\mathrm{W}_{0 \mathrm{JJ(tratamento} \mathrm{sol)}}-\mathrm{W}_{0 \mathrm{~J} \text { (tratamento sombra) }}\right.$ ), mostrando a banda-K. Os símbolos representam as espécies $C$. guianensis ( $\square)$ e $D$. odorata $(\Delta)$. 
Tabela 1 - Fluorescências inicial $\left(F_{0}\right)$, variável $\left(F_{v}\right)$ e máxima $\left(F_{m}\right)$, tempo de alcance da fluorescência máxima $\left(t_{F m}\right)$ e área entre a curva de fluorescência e $F_{m}$ de duas espécies arbóreas (fase juvenil) em dois ambientes de irradiância, com 45 dias de experimento.

\begin{tabular}{lcccccc}
\hline Espécie & Tratamento & $\mathrm{F}_{0}$ & $\mathrm{~F}_{\mathrm{v}}$ & $\mathrm{F}_{\mathrm{m}}$ & $\mathrm{t}_{\mathrm{Fm}}$ & Área \\
\hline \multirow{2}{*}{ C. guianensis } & Sol & $383 \pm 28 \mathrm{Aa}$ & $1113 \pm 52 \mathrm{Ba}$ & $1431 \pm 48 \mathrm{Ba}$ & $282 \pm 19 \mathrm{Ba}$ & $22600 \pm 3121 \mathrm{Ba}$ \\
& Sombra & $318 \pm 26 \mathrm{Ba}$ & $1383 \pm 84 \mathrm{Aa}$ & $1652 \pm 105 \mathrm{Aa}$ & $338 \pm 57 \mathrm{Aa}$ & $28040 \pm 2582 \mathrm{Aa}$ \\
\hline \multirow{2}{*}{ D. odorata } & Sol & $328 \pm 84 \mathrm{Aa}$ & $523 \pm 220 \mathrm{Bb}$ & $808 \pm 278 \mathrm{Bb}$ & $398 \pm 125 \mathrm{Aa}$ & $8720 \pm 2876 \mathrm{Bb}$ \\
& Sombra & $347 \pm 37 \mathrm{Aa}$ & $1053 \pm 47 \mathrm{Ab}$ & $1338 \pm 56 \mathrm{Ab}$ & $440 \pm 134 \mathrm{Aa}$ & $15520 \pm 3759 \mathrm{Ab}$ \\
\hline
\end{tabular}

Os valores são médias \pm desvios padrão. Médias seguidas da mesma letra maiúscula na vertical para tratamento, e da mesma letra minúscula na vertical, para espécie, não diferem estatisticamente pelo teste $t$ a $5 \%$ de probabilidade.

nitidamente, o efeito do tipo de estresse (Lichtenthaler et al., 1998). Assim, quando as plantas estáo sujeitas ao estresse pelo aumento da incidência de irradiância (p.ex.: exposição ao sol) a fase J torna-se mais pronunciada, fato este, observado para as espécies $C$. guianensis e $D$. odorata quando os valores do transiente OJIP são apresentados na forma de florescência variável relativa (Figura 2). Quando as plantas não estão sob efeito de determinado estresse não se verifica desvio positivo quando os dados são normalizados e exibidos na banda-K (Tomek et al., 2001; Oukarroum et al., 2007). A banda-K, normalmente é observada em plantas submetidas a estresse por elevação da temperatura (Guissé et al., 1995), contudo, sob aumento da incidência de irradiância também se verificou tal comportamento, como observado em experimento realizado por Gonçalves et al. (2007) e também verificado neste experimento (Figura 2). A presença da banda-K reflete uma inibição do complexo de evolução do oxigênio (OEC) e coincide com a limitação do lado doador do fotossistema II (Srivastava et al., 1997; Pospísil \& Dau, 2000; Tomek et al., 2001). Isto porque a transferência de elétrons do OEC para Yz é mais lenta do que a transferência de elétrons de P680 para a $Q_{A}$, surgindo a banda-K. Assim, qualquer condição de estresse que afeta a capacidade do lado doador implicará no surgimento da banda-K (Strasser, 1997).

\section{EFICIÊNCIA MÁXIMA DO FOTOSSISTEMA II E ÍNDICE DE DESEMPENHO}

Analisando-se os valores da eficiência máxima do fotossistema II (razão $\mathrm{F}_{\mathrm{v}} / \mathrm{F}_{\mathrm{m}}$ ) e o índice de desempenho $\left(\mathrm{IP}_{\mathrm{ABS}}\right)$, para as duas espécies estudadas, verificaram-se padróes similares de resposta em função do tempo de exposição aos tratamentos (dias) (Figura 4). Os valores médios de $\mathrm{F}_{\mathrm{v}} / \mathrm{F}_{\mathrm{m}}$ variaram de 0,82 a 0,83 para $C$. guianensis na sombra, ao passo que, para $D$. odorata, também na sombra, os valores variaram de 0,76 a 0,79 , valores estes que são de plantas estressadas, uma vez que valores abaixo de 0,83 para a razão $\mathrm{Fv} / \mathrm{Fm}$ são indicativos de presença de certo grau de estresse em muitas plantas (Björkman \& Demmig-Adams, 1987). Para as duas espécies estudadas, após 15 dias de experimento, as plantas exposta ao sol, exibiram valores significativamente menores de $\mathrm{F}_{\mathrm{v}} / \mathrm{F}_{\mathrm{m}}$, quando comparado com as plantas sob condiçóes de sombra. Quanto às respostas das plantas para $\mathrm{IP}_{\mathrm{ABS}}$, verificaram-se maiores valores para a espécie $C$. guianensis na sombra, quando comparada com $D$. odorata, também na sombra (2,35 a 2,87 e de 1,00 a 1,14, respectivamente). Para os indivíduos expostos ao sol das duas espécies estudadas os valores de $\mathrm{IP}_{\mathrm{ABS}}$ reduziram com o passar dos dias apresentando diferenças significativas. Resultados semelhantes verificados para os valores de $\mathrm{F}_{\mathrm{v}} / \mathrm{F}_{\mathrm{m}}$, após 15 dias de experimento (Figura 4). $\mathrm{O}$ valor médio de $\mathrm{PI}_{\mathrm{ABS}}$ para as plantas de C. guianensis expostas ao sol chegou a ser quase três vezes menor, quando comparado com os indivíduos na sombra após 15 dias de experimento. Ao passo que, para $D$. odorata, o valor médio do $\mathrm{IP}_{\mathrm{ABS}}$ para as plantas expostas ao sol foi duas vezes menor aproximadamente, quando comparado com os indivíduos expostos à sombra no mesmo período (Figura 4).

A significante diminuição nos valores de $\mathrm{F}_{\mathrm{v}} / \mathrm{F}_{\mathrm{m}}$ bem como nos valores de $\mathrm{IP}_{\mathrm{ABS}}$ indicam efeito de fotoinibiçáo e tais mudanças representam perda de eficiência fotoquímica pelas plantas (Thach et al., 2007). O índice de desempenho $\left(\mathrm{PI}_{\mathrm{ABS}}\right)$ tem sido considerado como um parâmetro mais sensível para a detecção e quantificação de estresse em plantas do que a resposta da eficiência máxima do fotossistema II $\left(\mathrm{F}_{\mathrm{v}} / \mathrm{F}_{\mathrm{m}}\right)$ (Christen et al., 2007; Oukarroum et al., 2007), ou seja, mais consistência para interpretação dos resultados tem sido obtida quando se avalia a resposta do fotossistema II para condiçóes estressantes usando o $\mathrm{PI}_{\mathrm{ABS}}$ do que apenas a razáo $\mathrm{F}_{\mathrm{v}} / \mathrm{F}_{\mathrm{m}}$. Isto porque esta variável relaciona a eficiência de absorçáo, captura e transferência de energia de excitação pelo fotossistema II proporcionando uma visão maior do grau de efeito do ambiente estressante (Gonçalves \& Santos Jr., 2005). Assim, pode-se notar, neste experimento, que ambas as espécies já se apresentavam sob efeito inibitório a pleno sol, após 15 dias de experimento, promovida pelo excesso de irradiância, e que a espécie $D$. odorata apresentou menor desempenho, quando comparada com C. guianensis. Alguns autores observaram que em plantios experimentais, plantas expostas a pleno sol de $D$. odorata e C. guianensis, apresentaram consideráveis taxas de sobrevivência e índices de crescimento em relação a outras espécies utilizadas em enriquecimento de capoeiras e, também, na recuperação de áreas degradadas na Amazônia (Alencar \& 
Araújo, 1980; Clay et al., 2000). Gonçalves et al. (2003) não observaram diferença para a razão $\mathrm{F}_{\mathrm{v}} / \mathrm{F}_{\mathrm{m}}$ em plantas de $D$. odorata crescendo sob ambientes sombreados $(0,79)$ e a pleno sol $(0,77)$ em plantios experimentais sobre áreas degradadas pela agricultura, porém, as concentraçóes de clorofila $a$ e $b$ diferiram entre os tratamentos de sol e de sombra. Marenco et al. (2001), não observaram diferenças na fotossíntese máxima $\left(\mathrm{A}_{\max }\right)$, condutância estomática (gs) e transpiração (E) em plantas de $D$. odorata com seis meses de idade crescendo em ambiente de sol, comparado com plantas crescendo em ambiente de sombra. Provavelmente esta espécie apresente uma boa estratégia de aclimatação nos diferentes ambientes luminosos em tempo relativamente prolongado, utilizando a irradiância de forma eficiente com reflexos diretos sobre as respostas das trocas gasosas, apesar de se ter observado neste experimento que, nos primeiros 45 dias após ser submetidas a maior exposição de irradiância, as plantas tenham sofrido certo grau de estresse.
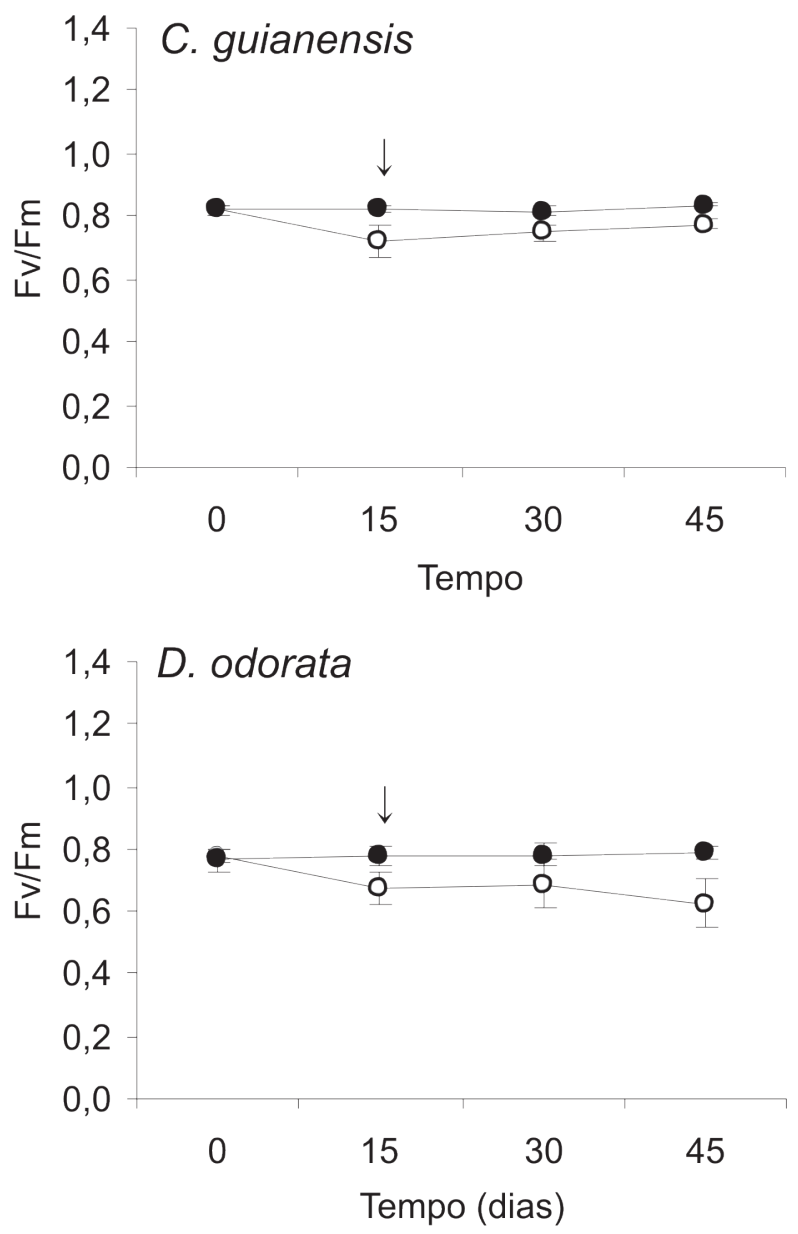

\section{FLUXO FENOMENOLÓGICO E RAZÕES DE FLUXOS}

Comparando-se as respostas (leituras inicial vs final) dos parâmetros do fluxo fenomenológico das duas espécies expostas ao sol, verificou-se reduçáo em RC/CS (15 e 34\% para $C$. guianensis e $D$. odorata, respectivamente), aumento em DI /CS (35\% para C. guianensis e 44\% para D. odorata), reduçâo em TR/CS (28\% para a espécie $D$. odorata) e redução em ET/CS ( 14 e 50\% para C. guianensis e D. odorata, respectivamente) (Figura 5A). Quando as respostas foram comparados entre os tratamentos (sombra $v$ sol), passados 45 dias após o início do experimento verificou-se nas plantas exposta ao sol redução em RC/CS (15 e 26\% para C. guianensis e D. odorata, respectivamente), aumento em ABS/CS (18\% para a espécie C. guianensis), aumento em DI /CS (63\% para C. guianensis e $69 \%$ para $D$. odorata), redução em $\mathrm{ET}_{\mathrm{o}} / \mathrm{CS}$ (42\% para a espécie D. odorata) (Figura 5B).
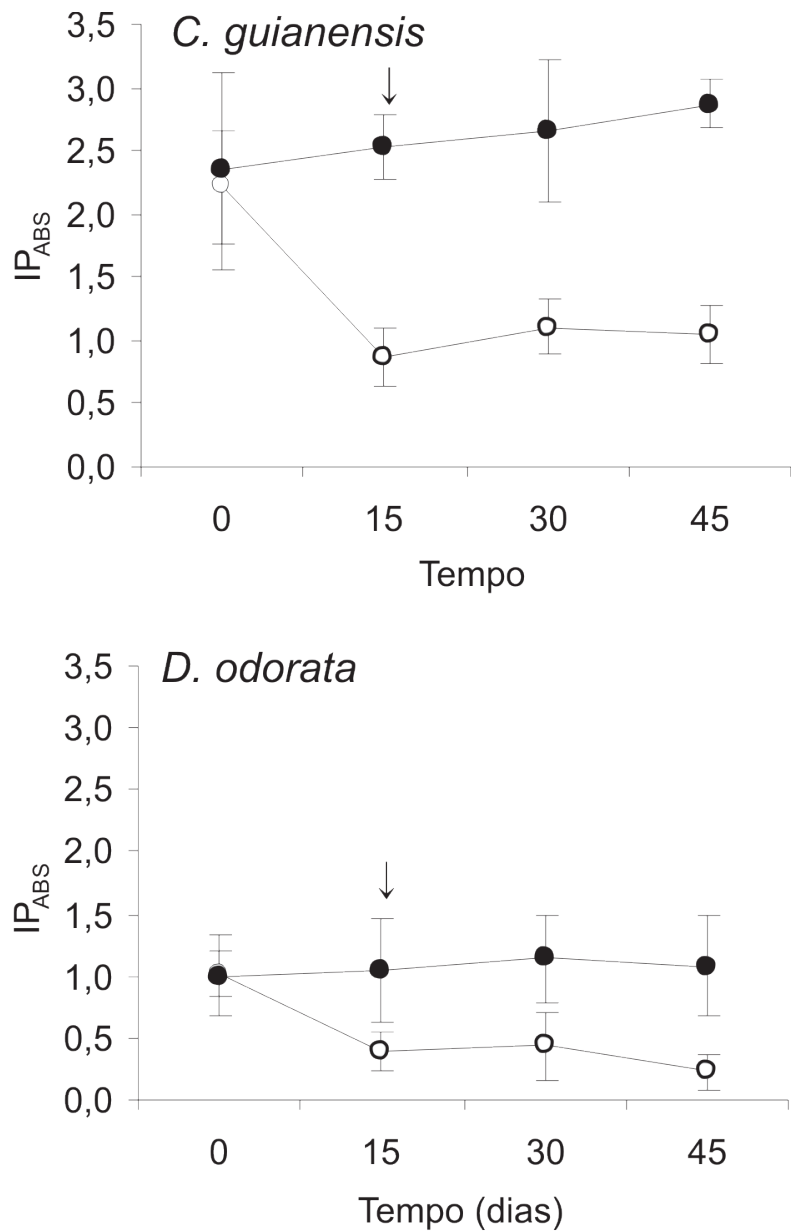

Figura 4 - Valores da razão $F_{V} / F_{m}$ e índice de desempenho ( $\left(P_{A B S}\right)$, em função do tempo (dias), de espécies arbóreas (fase juvenil) expostas à sombra (•) e ao sol (०). Os valores são médias e as linhas verticais indicam os desvios-padrão. A seta indica o início da diferença significativa entre os tratamentos pelo teste $t$ a $5 \%$ de probabilidade. 

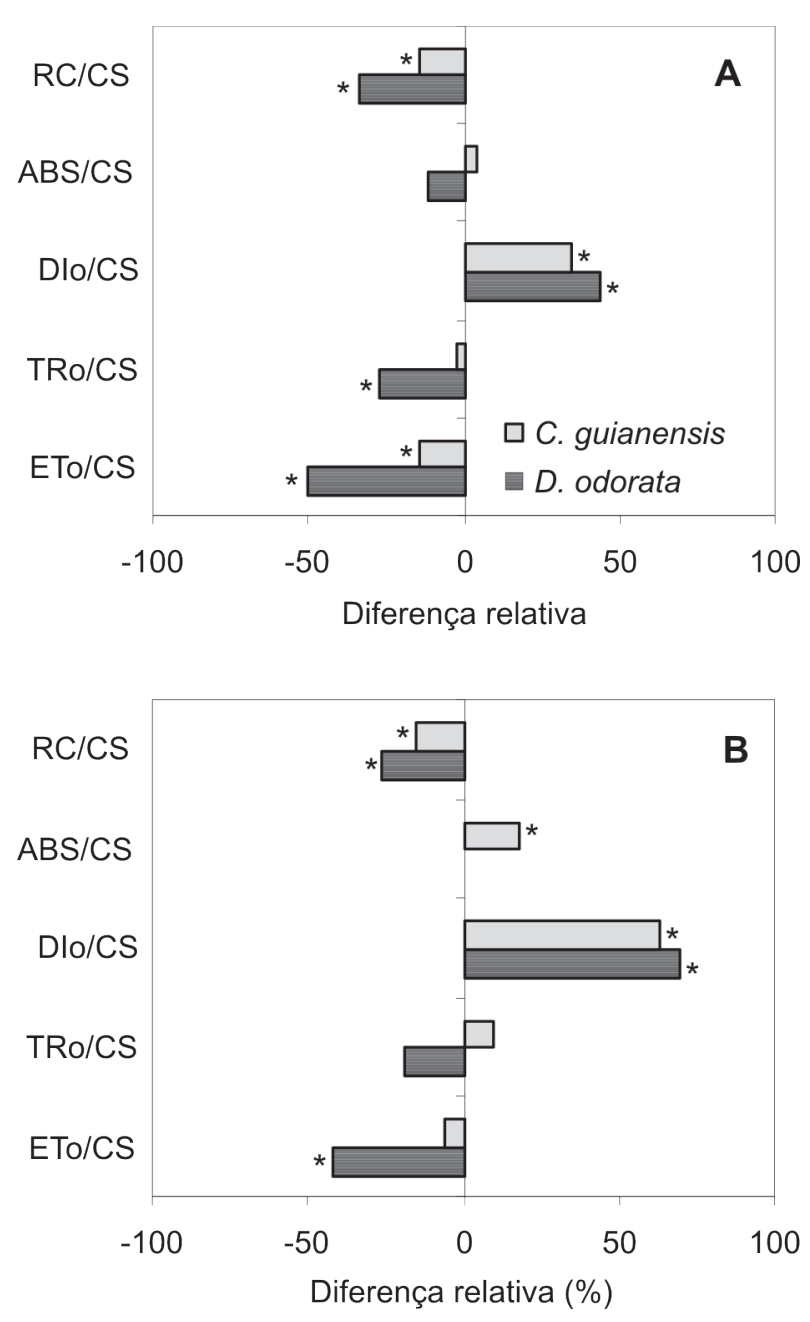

Figura 5 - Diferença relativa entre leitura final e leitura inicial do fluxo fenomenológico de duas espécies arbóreas (fase juveil) expostas somente ao sol (A) e, também, diferença entre exposição ao sol e à sombra passados 45 dias (B). RC/CS = densidade de centros de reação por secão transversal; $\mathrm{ABS} / \mathrm{CS}=$ número de fotóns absorvidos por seção transversal; $\mathrm{DI}_{\alpha} / \mathrm{CS}=$ Energia de dissipação por seção transversal; TR/CS = taxa máxima de energia bloqueada por seção transversal e; ET/CS = transporte de elétrons por seção transversal. 0 símbolo rasterisco repersenta diferença significativa entre as observações pelo teste $t$ a $5 \%$ de probabilidade.

Os elevados valores de $\mathrm{DI}_{\mathrm{o}} / \mathrm{CS}$ para as duas espécies expostas ao sol, tanto na comparaçáo da diferença da leitura final e leitura inicial, quanto na comparação da diferença entre os tratamentos de sol e sombra com 45 dias de exposição, indicam que grande parte da energia absorvida está sendo dissipada na forma de calor ou de fluorescência, o que implica déficit em RC/CS ativos, bem como, reflete redução em ET/ CS. O excesso de irradiância oferece mais energia do que pode ser utilizada pela planta criando uma situação na qual o mecanismo fotossintético não pode sustentar refletindo em perda na produtividade fotossintética (Thach et al., 2007).
Assim, os altos valores na dissipaçáo de energia podem ser explicados pelo fato da exposição à alta irradiância terem forçado as plantas para uma tentativa de se protegerem contra danos irreversíveis ao aparato fotossintético (Osmond et al., 1987), confirmando, assim, que as espécies estudadas são susceptíveis quando expostas à alta irradiância. Contudo, é possível notar, que, mesmo sob efeito inibitório, algumas espécies, como é o caso da C. guianensis, conseguem ter déficit de $\mathrm{ET}_{\mathrm{o}} / \mathrm{CS}$ pouco expressivo, quando comparado com a espécie $D$. odorata, tornando-a mais apta a situaçôes estressantes de aumento de irradiância.

Analisando-se as respostas das razóes de fluxos, verificouse que, para $\mathrm{TR}_{\mathrm{o}} / \mathrm{ABS}$, ambas as espécies apresentaram diferenças, quando comparadas entre tratamentos e, também, entre espécies (Figura 6), ou seja, para as plantas expostas à sombra, $C$. guianensis e $D$. odorata exibiram valores de 0,84 e 0,79 , respectivamente, enquanto que expostas ao sol exibiram valores bem abaixo $(0,78$ para $C$. guianensis e 0,63 para $D$. odorata). Entre as espécies, C. guianensis foi a mais eficiente, tanto exposta à sombra quanto ao sol. Contudo, grande parte dessa energia absorvida pelas duas espécies, de certa forma, teria sido dissipada em forma de calor ou fluorescência, uma vez que os valores de DI / $\mathrm{ABS}$ foram maiores para as plantas expostas ao sol, indicando que essas plantas já estariam sofrendo de fotoinibiçáo por excesso de irradiância. O que se pode notar, também, é que plantas de D. odorata expostas ao sol apresentaram maiores valores de DI / ABS confirmando seu baixo desempenho quando exposta a alta irradiância na fase juvenil. Conseqüentemente, em função da baixa absorção e elevada dissipação de energia, os valores de $\mathrm{ET}_{\mathrm{o}} / \mathrm{TR}_{\mathrm{o}}$ e $\mathrm{ET}_{\mathrm{o}} /$ ABS para a espécie $D$. odorata exposta ao sol, também, seriam os menores, quando comparado a mesma espécie exposta a sombra. Para ambas as espécies os maiores valores de $\mathrm{ET}_{\mathrm{o}} / \mathrm{TR}_{\mathrm{o}}$ e ET/ABS foram observados nas plantas expostas à sombra (Figura 6).

Gonçalves et al. (2007) estudando o comportamento de duas espécies arbóreas tropicais sob dois ambientes de irradiância (sombra e sol), também encontraram melhores respostas, relacionadas às razôes de fluxos, nos indivíduos expostos à sombra, bem como, atribuiu maior absorção, menor dissipação e melhor desempenho para a espécie $S$. macrophylla. Isto porque as plantas que estáo sob efeito de forte inibição por alta irradiância tendem a dissipar grande parte da energia, não direcionando essa energia de excitação para o transporte de elétrons com conseqüente diminuição da razão ET/ABS (Strasser et al., 1995). Uma explicação provável para esse efeito é uma considerável diminuição na densidade de centros de reação (RC/CS) (Figura 5B) mais do que, até certo ponto, a degradação da proteína $\mathrm{D} 1$, uma vez que a reduçáo deste parâmetro indica que parte dos centros de reação do fotossistema II está inativa (Hong \& Xu, 1999; 


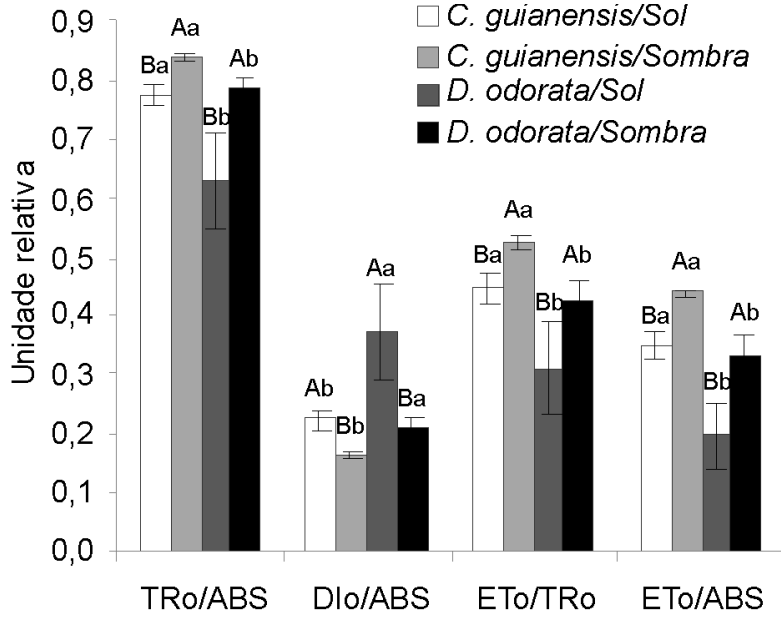

Figura 6 - Razões de fluxo de duas espécies arbóreas (fase juvenil) exposta à sombra e ao sol, com 45 dias de experimento. TR/ABS = Eficiência máxima do FSII; $\mathrm{DI}_{\mathrm{\rho}} / \mathrm{ABS}$ = eficiência máxima de excitação não fotoquímica; ET/TR = probabilidade da energia de excitação mover um elétron após a quinona $A ; E T / A B S=$ probabilidade de um fóton absorvido mover um elétron após a quinona $A$. Os valores são médias e as linhas verticais indicam os desviospadrão. Médias seguidas da mesma letra maiúscula, para tratamento, e da mesma letra minúscula, para espécie, não diferem significativamente pelo teste $t$ a $5 \%$ de probabilidade.

Li et al., 2007). Sob alta irradiância, freqüentemente, as folhas absorvem mais energia do que podem ser utilizadas pela fotossíntese, e para evitar uma situação de foto dano, dissipam o excesso da energia em forma de calor ou fluorescência, com conseqüente perda na produtividade fotossintética (Krause $e t$ al., 2001; Thach et al., 2007), fato que quase sempre leva a diminuição na incorporação de $\mathrm{CO}_{2}$ e diminuição nas fases de crescimento.

\section{CONCLUSÕES}

As espécies $C$. guianensis e $D$. odorata, expostas à alta irradiância, apresentaram considerável redução nas respostas dos transientes da fluorescência da clorofila $a$, bem como, dos valores dos parâmetros do fluxo fenomenológico e das razóes de fluxos. Contudo, apesar da exposiçáo à alta irradiância submeter ambas as espécies ao estado de estresse, C. guianensis apresentou melhores respostas a elevada irradiância, exibindo melhor desempenho do fotossistema II. Portanto, sob condições semelhantes de estresse lumínico, considera-se a $C$. guianensis como mais indicada para plantios de produção ou de recuperação de áreas degradadas na Amazônia.

\section{AGRADECIMENTOS}

Os autores agradecem ao Instituto Nacional de Pesquisas da Amazônia (MCT- INPA) e a toda a equipe do Laboratório de Fisiologia e Bioquímica Vegetal do INPA/LFBV pelo apoio intelectual e logístico dispensados para realização desta pesquisas. Os autores deste manuscrito também são gratos ao CNPq pelas concessóes das bolsas de iniciação científica, de mestrado e de produtividade.

\section{BIBLIOGRAFIA CITADA}

Alencar, J.C.; Araujo, V.C. 1980. Comportamento de espécies florestais amazônicas quanto à luminosidade. Acta Amazonica, 10: 435-444.

Boufleuer, Neuza Terezinha. Aspectos ecológicos de Andiroba (Carapa guianensis Aublet., Meliaceae), como subsídios ao manejo e conservação. 2004. Dissertação de Mestrado em Ecologia e Manejo de Recursos Naturais, Universidade Federal do Acre. Rio Branco. 84 pp.

Björkman, O.; Demmig-Adams, B. 1987. Photon yield of $\mathrm{O}_{2}$ evolution and chlorophyll fluorescence characteristics at 77 $\mathrm{k}$ among vascular plants of diverse origins. Planta, 170: 489504 .

Castro, Y., Fetcher, N., Fernandez, D.S. 1995. Chronic photoinhibition in seedlings of tropical trees. Physiologia Plantarum, 94: 560-565.

Christen, D.; Schönmann, S.; Jermini, M.; Strasser, R.J.; Défago, G. 2007. Characterization and early detection of grapevine (Vitis vinifera) stress responses to esca disease by in situ chlorophyll fluorescence and comparison with drought stress. Environmental and Experimental Botany, 60: 504-514.

Clay, J.W.; Sampaio, P.T.B.; Clement, C.R. 2000. Biodiversidade amazônica: exemplos e estratégias de utilizaçâo. Programa de Desenvolvimento Empresarial e Tecnológico, Manaus-AM. 409 pp.

Demmig-Adams, B.; Adams III, W.W. 1992. Photoprotection and other responses of plants to high light stress. Annual Reviews in Plant Physiology and Plant Molecular Biology, 43: 599-626.

Demmig-Adams, B., Adams, W.W.; Barker, D.H.; Logan, B.A.; Bowling, D.R.; Verhoeven, A.S. 1996. Using chlorophyll fluorescence to assess the fraction of absorbed light allocated to thermal dissipation of excess excitation. Physiologia Plantarum, 98: 253-264.

Denslow, J.S.; Schultz, J.C.; Vitousek, P.M.; Strain, B.R. 1990. Growth responses of tropical shrubs to treefall gap environments. Ecology, 71: 165-179.

Guissé, B.; Srivastava, A.; Strasser, R.J. 1995. The polyphasic rise of the chlorophyll $a$ fluorescence (O-K-J-I-P) in heat stressed leaves. Archive. Scientific Genève, 48: 147-160.

Gonçalves, J.F.C.; Fernandes, A.V.; Morais, R.R.; Melo, Z.L.O.; Santos Jr., U.M. 2003. Aspectos fisiológicos e bioquímicos de plantas da Amazônia, p. 89-101. In: Higuchi, N.; Santos, J.; Sampaio, P.T.B; Marenco, R.A.; Ferraz, J.; Sales, P.C.; Saito, M.; Matsumoto, S. (Eds) Projeto Jacaranda - Fase II: Pesquisas florestais na Amazônia central. INPA, Manaus, AM.

Gonçalves, J.F.C.; Santos Jr., U.M. 2005. Utilization of the chlorophyll a fluorescence technique as a tool for selecting tolerant species to environments of high irradiance. Brazilian Journal Plant Physiology, 17: 307-313. 
Gonçalves, J.F.C.; Santos Jr., U.M.; Nina Jr., A.R.; Chevreuil, L.R. 2007. Energetic flux and performance index in copaiba (Copaifera multijuga Hayne) and mahogany (Swietenia macrophylla King) seedlings under two irradiance environments. Brazilian Journal Plant Physiology, 19: 171-184.

Hong, S.S.; Xu, D.Q. 1999. Light-induced increase in initial chlorophyll fluorescence $\mathrm{F}_{\mathrm{o}}$ level and the reversible inactivation of PSII reaction centers in soybean leaves. Photosynthesis Research, 61: 269-280.

Krause, G.H.; Koroleva, O.Y.; Dalling, J.W.; Winter, K. 2001. Acclimation of tree seedling to excessive light in simulated treefall gaps. Plant, Cell and Environment, 24: 1345-1352.

Langenheim, J. H.; Osmond, C.B.; Brooks, A.; Ferrar, P.J. 1984. Photosynthetic responses to light in seedlings of selected Amazonian and Australian rainforest tree species. Oecologia, 63: $215-224$.

Lazár, D. 2006. The Polyphasic chlorophyll a fluorescence rise measured under high intensity of exciting light. Functional Plant Biology, 33: 9-30.

Lichtenthaler, H.K.; Wenzel, O.; Buschmann, C.; Gitelson, A. 1998. Plant stress detection by reflectance and fluorescence, p. 271-285. In: Annals New York Academy of Science.

Li, X.G.; Li, J.Y.; Zhao, J.P.; Xu, P.L.; He, Q.W. 2007. Xanthophyll cycle and inactivation of photosystem II reaction centers alleviating reducing pressure to photosystem I in morning glory leaves under short-term high irradiance. Journal of Integrative Plant Biology, 49: 1047-1053.

Long, S.P.; Humphries, S. Falkowski, P.G. 1994. Photoinhibition of photosynthesis in nature. Annual Reviews in Plant Physiology and Plant Molecular Biology, 45:633-662.

Lorenzi, H. 1998. Árvores brasileiras. Nova Odessa: Editora Plantarum. 222 pp.

Marenco, R.A.; Gonçalves, J.F.C.; Vieira, G. 2001. Leaf gas exchange and carbohydrates in tropical trees differing in successional status in two light environments in central Amazonia. Tree Physiology, 21: 1311-1318.

Osmond, C.B.; Austin, M.P.; Berry, J.A.; Billings, W.D.; Boyer, J.S.; Dacey, W.H.; Nobel, P.S.; Smith, S.D.; Winner, W.E. 1987. Stress physiology and the distribution of plants. BioScience, 37: 38-48.

Oukarroum, A.; El Madidi, S.; Schansker, G.; Strasser, R.J. 2007. Probing the responses of barley cultivars (Hordeum vulgare L.) by chlorophyll a fluorescence OLKJIP under drought stress and re-watering. Environmental and Experimental Botany, 60: 438-446.
Park, Y.; Chow, W.S.; Anderson, J.M.; Hurry, V.M. 1996. Differential susceptibility of photosystem II to light stress in light-acclimation pea leaves depends on the capacity for photochemical and nonradiative dissipation of light. Plant Science, 115: 137-149.

Pospíšil, P.; Dau, H. 2000. Chlorophyll fluorescence transiente of photosystem II membrane particles as a tool for studying photosynthetic oxygen evolution. Photosynthesis Research, 65: 41-52.

Santos Junior, U.M.; Gonçalves, J.F.C..; Feldpausch, T.R. 2006. Growth, leaf nutrient concentration and photosynthetic nutrient use efficiency in tropical species planted in degraded areas in central Amazonia. Forest Ecology and Management, 226: 299-309.

SAS Institute Inc., JMP Version 4.0, Cary, NC, USA: SAS Institute Inc., 2000.

Srivastava, A.; Guissé, B.; Greppin, H.; Strasser, R.J. 1997. Regulation of antenna structure and electron transport in photosystem II of Pisum sativum under elevated temperature probe by fast polyphasic chlorophyll a fluorescence transient: OKJIP. Biochimica et Biophysica Acta, 1320: 95-106.

Strasser, B. 1997. Donor side capacity of photosystem II probed by chlorophyll a fluorescence transients. Photosynthesis Research, 52: $147-155$.

Strasser, R.J.; Srivasatava, A.; Govindjee. 1995. Polyphasic chlorophyll a fluorescence transient in plants and cyanobacteria, Photochemistry and Photobiology, 61: 32-34.

Thach, L.B.; Shapcott, A.; Schmidt, S.; Critchley, C. 2007. The OJIP fast fluorescence rise characterizes Graptophyllum species and their stress responses. Photosynthesis Research, 94: 423-436.

Tomek, P.; Lazár, D.; Ilík, P; Naus, J. 2001. On intermediate steps between the $\mathrm{O}$ and $\mathrm{P}$ steps in chlorophyll a fluorescence rise measure at different intensities of exciting light. Australian Journal Plant Physiology, 28: 115-1160.

Tonini, H.; Oliveira Jr., M.M.C.; Schwengber, D. 2008. Crescimento de espécies nativas da Amazônia submetidas ao plantio no estado de Roraima. Ciência Florestal, 18: 151-158.

Walters, R.G. 2005. Towards an understanding of photosynthetic acclimation. Journal of Experimental Botany, 56: 435-447.

Recebido em: 23/10/2008

Aceito em: 12/05/2009 
